\title{
Generalized Płonka Sums and Products
}

\author{
Marek Zawadowski
}

Received: 28 October 2012 / Accepted: 28 April 2013 / Published online: 7 December 2013

(C) The Author(s) 2013. This article is published with open access at Springerlink.com

\begin{abstract}
We give an abstract categorical treatment of Płonka sums and products using lax and oplax morphisms of monads. Płonka sums with sup-semilattices as arities were originally defined as operations on algebras of regular theories. It turns out that even more general operations are available on the categories of algebras of semi-analytic monads. Their arities are the categories of the regular polynomials over any sup-semilattice, i.e. any algebra for the terminal semi-analytic monad. We also show that similar operations can be defined on the category of algebras of any analytic monad. This time we can allow the arities to be the categories of linear polynomials over any commutative monoid, i.e. any algebra for the terminal analytic monad. There are also dual operations of Płonka products. They can be defined on Kleisli categories of commutative monads.
\end{abstract}

Keywords Analytic monad - Semi-analytic monad · Morphism of monads · Płonka sum

Mathematics Subject Classifications (2010) $03 \mathrm{D} 35 \cdot 03 \mathrm{C} 05 \cdot 03 \mathrm{G} 30 \cdot 18 \mathrm{C} 10 \cdot 18 \mathrm{C} 15$

\section{Introduction}

When dealing with a specific kind of categories one of the first questions we might ask is 'What kind of limits and colimits they have?'. Both operations are defined via universal properties, thus, if they exist, they are unique up to an isomorphism. However, in many circumstances we have interesting operations on a category which are not given by universal properties, yet in a given context they might be very useful. For example, one can equip a category with tensor product making it into a monoidal category $[3,13,14]$. Such a monoidal structure, if it exists, does not need to be unique in any sense. If we deal with

Dedicated to George Janelidze on the occasion of his 60th birthday

M. Zawadowski $(\bowtie)$

Instytut Matematyki, Uniwersytet Warszawski, ul. S.Banacha 2, 00-913 Warszawa, Poland

e-mail: zawado@mimuw.edu.pl 
categories of models of first order theories, we can equip them with ultraproduct operations [12]. These ultraproduct operations, even if they are not given by universal properties, have received a very fruitful categorical treatment (c.f. $[15,16])$ and proved to be useful in definability theory of first order logic, see [17, 24].

The aim of this paper is to give a categorical treatment of Płonka sums (c.f. [19]). Originally, Płonka sums were defined as operations on categories of algebras of regular equational theories with arites being semilattices. They are related to the (strong) sup-semilattice decomposition of semigroups $[8,23]$. The latter perspective though puts more emphasis on decomposing of a given algebra into simpler pieces rather than building more complicated algebras from simpler ones. If $L$ is a sup-semilattice considered as a (posetal) category and $\mathcal{R}$ is a monad on Set corresponding to a regular equational theory, i.e. a semi-analytic monad (c.f. [22]), then $L$-indexed Płonka sum is a functor $\bigsqcup_{L}: \mathcal{E} \mathcal{M}(R)^{L} \longrightarrow \mathcal{E} \mathcal{M}(R)$, i.e. an operation on the Eilenberg-Moore category of the monad $\mathcal{R}$. For $F: L \rightarrow \mathcal{E} \mathcal{M}(\mathcal{R})$ the operation associates an $\mathcal{R}$-algebra whose universe is the coproduct $\bigsqcup_{l \in L} F(l)$ (in Set) and we define the operations on this universe using the transition homomorphisms $F\left(l \leq l^{\prime}\right): F(l) \rightarrow F\left(l^{\prime}\right)$ between the $\mathcal{R}$-algebras. To calculate the value of an $n$-ary operation $f$ on elements $a_{i} \in F\left(l_{i}\right)$ for $i=1, \ldots, n$, we first move those elements $a_{i}$ to a common place, that is to the algebra $F\left(\bigvee_{i} l_{i}\right)$ applying $\mathcal{R}$ homomorphisms $F\left(l_{i} \leq \bigvee_{i} l_{i}\right)$ to elements $a_{i}$ and then we apply the operation $f$ to those moved elements inside the $\mathcal{R}$-algebra $F\left(\bigvee_{i} l_{i}\right)$.

In categorical terms, Płonka sums are functors induced by lax morphisms of monads. If $(\mathcal{T}, \eta, \mu)$ is a monad on Set and $C$ is a small category, then we have a monad $(\hat{\mathcal{T}}, \hat{\eta}, \hat{\mu})$ on Set ${ }^{C}$, the lift of the monad $\mathcal{T}$ to $\mathrm{Set}^{C}$, defined by composition:

$$
\hat{\mathcal{T}}(F)=\mathcal{T} \circ F, \quad \hat{\eta}_{F}=\eta_{F}: F \rightarrow T \circ F, \quad \hat{\mu}_{F}=\mu_{F}: T^{2} \circ F \rightarrow T \circ F
$$

where $\hat{\eta}_{F}$ is the component of the natural transformation $\hat{\eta}$ at a functor $F: C \rightarrow$ Set and $\eta_{F}$ is the wiskering of the natural transformation $\eta: 1_{\text {Set }} \rightarrow \mathcal{T}$ along the functor $F$; the same applies to the definition of $\hat{\mu}$.

With this Płonka sum of arity $C$ is a functor induced by lax morphisms of monads $\left(\bigsqcup_{C}, \phi\right): \hat{\mathcal{T}} \rightarrow \mathcal{T}$ whose functor part is the coproduct operation

$$
\bigsqcup_{C}: \text { Set }^{C} \longrightarrow \text { Set }
$$

so that

$$
F \mapsto \coprod_{c \in C} F(c)
$$

The problem is what kind of monads $\mathcal{T}$ and what kind of categories $C$ we should consider to get such lax morphism of monads. We show that in case of semi-analytic monads the natural choice for these arities are the categories of regular polynomials over sup-semilattices, i.e. the algebras for the terminal semi-analytic monads. In case of analytic monads the natural choice for these arities are the categories of linear polynomials over commutative monoids, i.e. the algebras for the terminal analytic monads. There are also natural infinitary generalizations of these. In each case, the category of the monads in question (analytic, semi-analytic, and their generalizations) is a coreflexive subcategory in the category of all monads on Set and the Płonka sums have arities being categories of some kind of polynomials over the algebras for the terminal monad in this subcategory. Such Płonka sums can be considered an additional structure on a category. We show that the preservation of Płonka sums by a functor between categories of algebras ensures that the morphism of monads that induced it belonged to the appropriate subcategory of monads (c.f. Theorems 5.5, 5.6, 7.2). 
The reason why Płonka sums work well for semi-analytic monads is that in the corresponding theories there is a good notion of an occurrence of a variable in a term. By this we mean that if two terms are equivalent modulo such a theory, the same variables occur in both of them. For analytic monads Płonka sums work, in a sense, even better (i.e. the arities can be categories of linear polynomials over monoids). This is due to the fact that in the corresponding equational theories there is a good notion of a specific occurrence of a variable in a term. This means that if two terms are equivalent modulo such a theory, each variable occurs in each of them the same number of times and each occurrence of a variable on one side of an equation can be related to an occurrence of the same variable on the other side. Moreover, if in such equations we substitute any term just for the related occurrences of a variable on both sides, then we will still have an equation.

As we said, the terminal objects in categories of analytic and semi-analytic monads play an important role. They have yet another property with respect to other monads in the respective categories. The commutative monoid monad distributes over any analytic monad in a canonical way and the sup-semilattice monad distributes over any semi-analytic monad in a canonical way, as well.

Looking at Płonka sums from that abstract point of view, it is clear that there can be, at least in principle, dual operations of Płonka products on the categories of Kleisli algebras for monads. Such monads seem to be even more rare, but surprisingly this kind of phenomena did already appear in the literature (although dressed in a different setting). We will show that for any commutative monad $\mathcal{T}$ there are operations of Płonka product with arities being finite sets. Note that the category of finitary commutative monads is a reflective subcategory of the category of all finitary monads.

One can trace origin of Płonka sum in the construction of the (strong) semi-lattice decomposition of semi-groups [23]. Since its introduction in 1967, Płonka sum generated a substantial work including some specializations like [6] and generalizations [7]. The generalization in [7] is considering transition morphisms between algebras that are more general than homomorphisms, yet give rise to an operation with interesting properties. However the author is unaware of any categorical treatment of the notion in the literature.

The paper is organized as follows. In Section 2, we describe some subcategories of endofunctors on Set and categories of monads over such categories. The only new result in this section is the characterization of the infinitary generalizations of the category of semianalytic functors and monads. In Section 3, we describe the general setup for both Płonka sums and products. The categories of regular and linear polynomials over algebras for semianalytic and analytic monads are described in Sections 4 and 6, respectively. Płonka sums on the categories of algebras for semi-analytic monads and their infinitary generalizations are described in Section 5. Płonka sums on the categories of algebras for analytic monads and their infinitary generalizations are described in Section 7. In Section 8, some examples of concrete Płonka sums are presented. In Sections 9, Płonka products are discussed shortly. Finally in Section 10, we discuss some distributive laws and the properties of the composed monads.

\section{Preliminary Notions and Notation}

In the paper we shall use category theory as well as 2-category theory language. We use the theory of monads in the abstract setting of 2-categories, including morphisms of monads, distributive laws, Eilenberg-Moore and Kleisli objects, and the theory of monoidal categories and monoidal monads (c.f. [2, 4, 11, 13, 20]). 
$\omega$ denotes the set of natural numbers. A cardinal number is the least ordinal of the given cardinality. Thus any $n \in \omega$ is a cardinal number. If $\alpha$ is a cardinal number, then $(\alpha]=$ $\{1, \ldots, \alpha\} . S_{\alpha}$ is the symmetric group, i.e. the group of permutations of the set $(\alpha]$. We write $X^{\alpha}$ for the set of functions from $(\alpha]$ to $X$. The set $X^{\alpha}$ is interpreted as $X^{(\alpha]}$ and it has a (natural) right action of the permutations group $S_{\alpha}$ by composition. When $S_{\alpha}$ acts on the set $A$ on the right and on the set $B$ on the left, the set $A \otimes_{\alpha} B$ is the usual tensor product of $S_{\alpha}$-sets.

\section{Coreflective Subcategories of the Categories of Monads}

The categories of (finitary) analytic monads AnMnd and semi-analytic monads SanMnd are (non-full) subcategories of the category Mnd of finitary monads on Set. They are categories of monoids in monoidal categories An, San, End of analytic, semi-analytic, and all finitary endo-functors on Set, respectively. The analytic functors were introduced in [9], the semi-analytic monads were introduced in [18] under the name of collection monads. The categories An, San, AnMnd and SanMnd were studied extensively in [21, 22].

Both kinds of functors have two characterizations: one abstract and one very concrete. Let $\mathbb{S}$ be the skeleton of the category of finite sets and surjection whose objects are sets $\{1, \ldots, n\}$, for $n \in \omega, \mathbb{B}$ its subcategory with the same objects whose morphisms are bijections only.

An analytic functor in An is a finitary endofunctor on Set weakly preserving wide pullbacks. More concretely, $\mathcal{A}$ is an analytic functor iff there is a functor $A: \mathbb{B} \rightarrow$ Set and a natural isomorphism

$$
\mathcal{A}(X) \cong \sum_{n \in \omega} X^{n} \otimes_{n} A_{n}
$$

where $X$ is a set and $X^{n} \otimes_{n} A_{n}$ is the quotient of the product of a right $S_{n}$-set $X^{n}$ with the left $S_{n}$-set $A_{n}$.

A semi-analytic functor San is a finitary endofunctor on $S e t$ preserving pullbacks along monos. More concretely, $\mathcal{R}$ is a semi-analytic functor iff there is a functor $R: \mathbb{S} \rightarrow$ Set and a natural isomorphism

$$
\mathcal{R}(X) \cong \sum_{n \in \omega}\left[\begin{array}{l}
X \\
n
\end{array}\right] \otimes_{n} R_{n}
$$

where $X$ is a set and $\left[\begin{array}{l}X \\ n\end{array}\right] \otimes_{n} R_{n}$ is the quotient of the product of a right $S_{n}$-set $\left[\begin{array}{c}X \\ n\end{array}\right]$ of injections from ( $n$ ] to $X$ with the left $S_{n}$-set $R_{n}$. For more details see [22]. The categories An and San are coreflective in Mnd. If $\mathcal{T}$ is a finitary endofunctor in End, then its reflection in An is, for any set $X$, given by

$$
\operatorname{an}(\mathcal{T})(X)=\sum_{n \in \omega} X^{n} \otimes_{n} \mathcal{T}(n)
$$

and in San is given by

$$
\operatorname{san}(\mathcal{T})(X)=\sum_{n \in \omega}\left[\begin{array}{l}
X \\
n
\end{array}\right] \otimes_{n} \mathcal{T}(n)
$$

The categories An and San have terminal objects, the functors

$$
\sum_{n \in \omega} X^{n} \otimes_{n} 1 \quad \text { and } \quad \sum_{n \in \omega}\left[\begin{array}{l}
X \\
n
\end{array}\right] \otimes_{n} 1
$$


respectively. The morphisms in An are weakly cartesian natural transformations and the morphisms in San are semi-cartesian natural transformations, i.e. the natural transformations such that the commuting naturality squares for injections are pullbacks.

The categories An, San, End are (strictly) monoidal and the inclusion functors An $\rightarrow$ San $\rightarrow$ End are strictly monoidal functors. Thus the right adjoints $a n$ and san are lax monoidal functors. In particular, they preserve terminal object and so do the induced right adjoint functors between the categories of monoids, i.e. suitable monads. The terminal monad in AnMnd is the monad for commutative monoids $\mathcal{C}$, and the terminal monad in SanMnd is the finite power-set monad i.e. the monad for sup-semilattices $\mathcal{L}$.

These concepts have various infinitary generalizations. We can replace the category of finitary endofunctors End by the category $\mathbf{E n d}_{\kappa}$ of $\kappa$-accessible endofunctor, where $\kappa$ is an infinite regular cardinal or $\infty$ (End $\mathbf{E}_{\infty}$ is the category of all accessible functors), or even the category MND of all endofunctors on Set. For each category End $_{\kappa}$, End $_{\infty}$, END there is a corresponding (concrete) notion of analytic and semi-analytic functor $\left(\mathbf{A} \mathbf{n}_{\kappa}, \mathbf{A n} \mathbf{n}_{\infty}, \mathbf{A N}\right.$, $\left.\mathbf{S a n}_{\kappa}, \operatorname{San}_{\infty}, \mathbf{S A N}\right)$ and $\operatorname{monad}\left(\mathbf{A n M n d}_{\kappa}, \operatorname{AnMnd}_{\infty}, \mathbf{A N M N D} \operatorname{SanMnd}_{\kappa}, \operatorname{SanMnd}_{\infty}\right.$, SANMND).

Let $\mathbb{B}_{\kappa}$ denote the category of cardinal numbers smaller than $\kappa$ with bijections. An object $\mathcal{A}$ of $\mathbf{A} \mathbf{n}_{\kappa}$ is, up to an isomorphism, given by a functor $A: \mathbb{B}_{\kappa} \rightarrow$ Set so that for any set $X$

$$
\mathcal{A}(X) \cong \sum_{\alpha \in \operatorname{Card}_{\kappa}} X^{\alpha} \otimes_{\alpha} A_{\alpha}
$$

where $\operatorname{Card}_{\kappa}$ is the set of objects of $\mathbb{B}_{\kappa}$, i.e. the set of all cardinal numbers smaller than $\kappa$. The morphisms in $\mathbf{A n}_{\kappa}$ are those induced as above by morphisms in $\operatorname{Set}^{\mathbb{B}_{\kappa}}$, i.e. the categories $\mathbf{A n}_{\kappa}$ and $\mathrm{Set}^{\mathrm{B}_{\kappa}}$ with $\kappa$ regular are equivalent. It can be shown that the objects of $\mathbf{A} \mathbf{n}_{\kappa}$ are $\kappa$-accessible functors that preserve wide pullbacks (c.f. [1]), and that the morphisms in this category are weakly cartesian natural transformations. The categories $\mathbf{A} \mathbf{n}_{\infty}$ and $\mathbf{A N}$ are the same and it is the 'sum' of all the categories $\mathbf{A} \mathbf{n}_{\kappa}$ with $\kappa$ regular cardinal.

The infinitary generalizations of semi-analytic functors haven't been considered yet, but they are also quite natural. Let $\mathbb{S}_{\kappa}$ denote the category of cardinal numbers smaller than $\kappa$ with surjections, $\mathbb{S}_{\infty}$ the category of all cardinal numbers with surjections. An object $\mathcal{R}$ of $\mathbf{S a n}_{\kappa}$ is, up to an isomorphism, given by a functor $R: \mathbb{S}_{\kappa} \rightarrow$ Set so that for any set $X$

$$
\mathcal{R}(X) \cong \sum_{\alpha \in \text { Card }_{\kappa}}\left[\begin{array}{l}
X \\
\alpha
\end{array}\right] \otimes_{\alpha} R_{\alpha}
$$

The morphisms in $\mathbf{S a n}_{\kappa}$ are induced by morphisms in $\operatorname{Set}^{\mathrm{S}_{\kappa}}$, so that the categories $\mathbf{S a n}_{\kappa}$ and $\mathrm{Set}^{\mathrm{S}_{\kappa}}$ with $\kappa$ regular cardinal are equivalent.

The category $\operatorname{San}_{\infty}$ is the 'sum' of all the categories $\mathbf{S a n}_{\kappa}$ with $\kappa$ regular cardinal. An object $\mathcal{R}$ of $\mathbf{S A N}$ is, up to an isomorphism, given by a functor $R: \mathbb{S}_{\infty} \rightarrow$ Set, so that for any set $X$

$$
\mathcal{R}(X) \cong \sum_{\alpha \in \text { Card }}\left[\begin{array}{l}
X \\
\alpha
\end{array}\right] \otimes_{\alpha} R_{\alpha}
$$

Card is the class of all cardinal numbers. Note that this sum is well defined as for $\alpha>$ $\operatorname{card}(X)$ the set $\left[\begin{array}{l}X \\ \alpha\end{array}\right]$ of injections from $(\alpha]$ to $X$ is empty. The categories $\mathbf{S a n}_{\infty}$ and $\operatorname{Set}^{\mathbb{S}_{\infty}}$ are equivalent. The following theorem provides a characterization of the above categories.

Theorem 2.1 The objects of the category $\mathbf{S a n}_{\kappa}$, where $\kappa$ is either a regular cardinal or $\infty$, are $\kappa$-accessible functors that preserve pullbacks along monos and wide pullbacks of 
monos. The objects of the category SAN are functors that preserve pullbacks along monos and wide pullbacks of monos. The morphisms in these categories are semi-cartesian natural transformations.

Proof We shall prove the characterization for $\mathbf{S a n}_{\kappa}$ where $\kappa$ is any regular cardinal. The other two cases are similar and can be easily deduced.

Thus, by an argument analogous to the one given in the proof of Proposition 2.1 of [22], we need to identify the image of Kan extension functor $\mathrm{Set}^{\mathrm{S}_{\kappa}} \rightarrow$ END. This is a 'lift' of the proof of Theorem 2.2 of [22] from $\kappa=\omega$ to any regular cardinal $\kappa$. On one hand, if an endofunctor $\mathcal{R}$ on Set is defined by the formula (1), then it easy to see that it preserves wide pullbacks of monos. On the other hand, if an endofunctor $F$ on Set is $\kappa$-accessible and preserves wide pullbacks of monos, then for any set $X$ and any $x \in F(X)$ there is the least cardinal $\alpha$ necessarily smaller then $\kappa$ and the least subobject $F:(\alpha] \longrightarrow X$ and $y \in F(\alpha]$ such that $F(f)(y)=x$. With these observations the rest can be easily deduced from the proof of Theorem 2.2. in [22].

Having the above characterization it is clear now that all the categories of functors considered above are closed under composition of their objects. For the explicite formulas for the coefficient functors of the composed functors in categories An and San the reader can consult $[21,22]$. Thus we have described a diagram of (strictly monoidal) categories and functors.

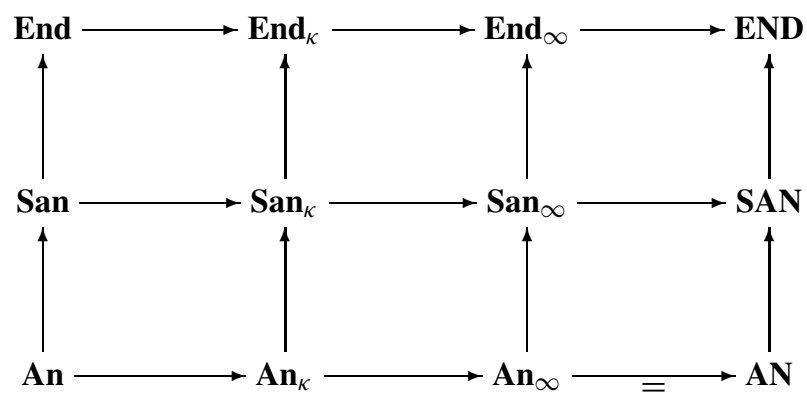

All the functors are (strictly monoidal) inclusions. Horizontal inclusions are full. All the categories but $\mathbf{A n} \mathbf{n}_{\infty}=\mathbf{A N}$ and $\mathbf{S a n} \mathbf{n}_{\infty}$ are coreflective in all the categories that contain them. All the categories but $\mathbf{A} \mathbf{n}_{\infty}=\mathbf{A N}$ and $\mathbf{S a n} \mathbf{n}_{\infty}$ have terminal objects which are the values of the right adjoints to the inclusion on the terminal object in END.

The above diagram lifts to the diagram of monoids in those categories and we get the following categories of monads with functors being again inclusions

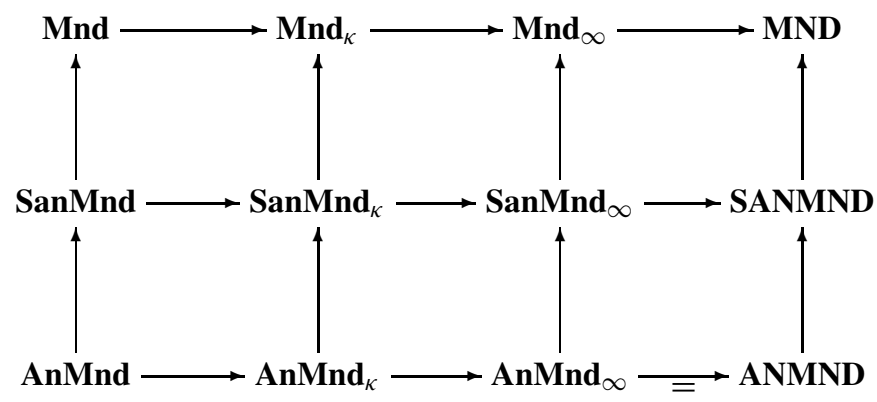


If a monoidal category has a terminal object 1 , then there is a unique structure of a monoid on 1 and this monoid is the terminal monoid. Therefore all the above categories of monads but $\mathbf{A n M n d}_{\infty}=\mathbf{A N M N D}$ and SanMnd $\mathbf{S}_{\infty}$ have terminal objects. The terminal object in AnMnd is the monad for commutative monoids and the terminal object in $\mathbf{A n M n d}_{\kappa}$ is its 'less than $\kappa$ ' version. The terminal object in SanMnd is the monad for sup-semilattices and the terminal object in $\mathbf{S a n M n d}_{\kappa}$ is its 'less than $\kappa$ ' version. The terminal object in SANMND is the power-set monad i.e. the monad for sup-lattices.

\section{A General Setup for Płonka Sums and Products}

Let CAT denote the 2-category of possibly large categories, functors, and natural transformations. Let $\mathbf{M N D}_{\text {lax }}$ and $\mathbf{M N D}_{\text {oplax }}$ denote the 2-categories of monads in CAT as 0-cells, with lax and oplax morphism of monads as 1-cells, and with transformations of lax and oplax morphism of monads as 2-cells, respectively. Let $\mathcal{E M}: \mathbf{M N D}_{\text {lax }} \longrightarrow$ CAT denote the 2-functor of Eilenberg-Moore object, and $\mathcal{K}: \mathbf{M N D}_{\text {oplax }} \longrightarrow$ CAT denote the 2-functor of Kleisli object (c.f. [20]).

Let $\mathcal{S}=(\mathcal{S}, \eta, \mu)$ be a monad on a complete and cocomplete category $\mathcal{A}$. If $C$ is a small category, then $\mathcal{S}$ lifts to a monad $\hat{\mathcal{S}}$ on the functor category $\mathcal{A}^{C}$ (see introduction for details of the definition of $\hat{\mathcal{S}}$ ) so that the diagonal functor

$$
\delta_{\mathcal{A}}: \mathcal{A} \longrightarrow \mathcal{A}^{C}
$$

is a strict morphism of monads $\mathcal{S} \rightarrow \hat{\mathcal{S}}$. As $\delta_{\mathcal{A}}$ has both adjoints $\operatorname{Colim}_{\mathcal{A}, C} \dashv \delta_{\mathcal{A}} \dashv$ $\operatorname{Lim}_{\mathcal{A}, C}$, it induces an oplax morphism of monads $\left(\operatorname{Colim}_{\mathcal{A}, C}, \psi_{C}\right): \hat{\mathcal{S}} \rightarrow \mathcal{S}$ and lax morphism of monads $\left(\operatorname{Lim}_{\mathcal{A}, C}, \psi_{l}\right): \hat{\mathcal{S}} \rightarrow \mathcal{S}$.

The exponentiation 2-functor $(-)^{C}:$ CAT $\rightarrow$ CAT commutes with Eilenberg-Moore objects in CAT. Thus the functor $\mathcal{E} \mathcal{M}\left(\delta_{\mathcal{A}}\right)$ between Eilenberg-Moore categories induced by the strict morphism of monads $\delta_{\mathcal{A}}$ factorizes via the diagonal functor $\delta_{\mathcal{E} \mathcal{M}(\mathcal{S})}$ as follows

$$
\mathcal{E} \mathcal{M}(\mathcal{S}) \stackrel{\delta_{\mathcal{E M}}(\mathcal{S})}{\longrightarrow} \mathcal{E M}(\mathcal{S})^{C} \stackrel{\simeq}{\longrightarrow} \mathcal{M}(\hat{\mathcal{S}})
$$

As $\left(\operatorname{Lim}_{C}, \psi_{l}\right)$ is the right adjoint to $\delta_{\mathcal{A}}$ in $\mathbf{M N D}_{\text {lax }}$, the functor $\mathcal{E} \mathcal{M}\left(\operatorname{Lim}_{C}, \psi_{l}\right)$ is the right adjoint to $\mathcal{E} \mathcal{M}\left(\delta_{\mathcal{A}}\right)$ in $\mathbf{C A T}$ and it factorizes as

$$
\mathcal{E} \mathcal{M}(\hat{\mathcal{S}}) \stackrel{\simeq}{\longrightarrow} \mathcal{E M}(\mathcal{S})^{C} \stackrel{\overline{\operatorname{Lim}}_{C}}{\longrightarrow} \mathcal{E} \mathcal{M}(\mathcal{S})
$$

$\overline{\operatorname{Lim}}_{C}$ is nothing but the functor of taking limits of $\mathcal{S}$-algebras indexed by the small category $C$. This is a way we can explain why categories of Eilenberg-Moore algebras are complete whenever the categories over which they are defined are.

As it is well-known, the situation with Kleisli algebras is very different. Typically, the Kleisli category is not cocomplete even if the category over which it is defined is. ${ }^{1}$ This is

\footnotetext{
${ }^{1}$ For example, for finitary monads in Set Kleisli categories have all (small) coproducts, but almost never coequalizers. Any Eilenberg-Moore algebra is a coequalizer of a (canonical) parallel pair of morphisms between free algebras.
} 
due to the fact that the exponentiation 2-functor $(-)^{C}:$ CAT $\rightarrow$ CAT does not commute with Kleisli objects, in general. It does if the index category $C$ is discrete. So the above argument can be repeated only in case $C$ is a discrete category, i.e. in such case the functor $\mathcal{K}\left(\operatorname{Colim}_{\mathcal{A}, C}, \psi_{c}\right)$, the left adjoint to $\mathcal{K}\left(\delta_{\mathcal{A}}\right)$, factorizes as

$$
\mathcal{K}(\hat{\mathcal{S}}) \stackrel{\simeq}{\longrightarrow} \mathcal{K}(\mathcal{S})^{C} \stackrel{\overline{\operatorname{Colim}}_{C}}{\longrightarrow} \mathcal{K}(\mathcal{S})
$$

and $\overline{\operatorname{Colim}}_{C}$ is the usual coproduct of free algebras. This is a way we can explain why categories of Kleisli algebras have coproducts, but not all colimits in general, whenever the categories over which they are defined have either coproducts or even all colimits.

The natural transformation $\psi_{c}$ is a natural isomorphism i.e. $\left(\operatorname{Colim}_{C}, \psi_{c}\right)$ is a strong morphism of monads iff $\mathcal{S}$ preserves $C$ indexed colimits. If $\mathcal{A}=\operatorname{Set}, \mathcal{S}$ is finitary and this holds even for (finite) discrete categories $C$, it implies that all the operations in the equational theory corresponding to the monad $\mathcal{S}$ are unary. Thus such monads are very rare and mostly uninteresting. However, if a monad $\mathcal{S}$ has some additional good properties (like being analytic or semi-analytic on $\mathcal{A}=S e t$ ), it may happen that for some small category $C$ the functor $\bigsqcup_{C}: \mathcal{A}^{C} \longrightarrow \mathcal{A}$, the composition of functors

$$
\mathcal{A}^{C} \stackrel{i^{*}}{\longrightarrow} \mathcal{A}^{o b(C)} \stackrel{\amalg}{\longrightarrow} \mathcal{A}
$$

induced by functors between small categories $C \stackrel{i}{\longleftarrow} o b(C) \stackrel{!}{\longrightarrow} 1$, can be equipped with a natural transformation $\lambda_{C}: \mathcal{S} \circ \bigsqcup_{C} \longrightarrow \bigsqcup_{C} \circ \hat{\mathcal{S}}$ so that $\left(\bigsqcup_{C}, \lambda_{C}\right): \hat{\mathcal{S}} \rightarrow \mathcal{S}$ is a lax morphism of monads. Each such lax morphism of monads induces in turn an operation on the category of Eilenberg-Moore algebras

$$
\bigsqcup_{C}: \mathcal{E} \mathcal{M}(\mathcal{S})^{C} \cong \mathcal{E} \mathcal{M}(\hat{\mathcal{S}}) \longrightarrow \mathcal{E} \mathcal{M}(\mathcal{S})
$$

In a special case these operations are what is called Płonka sum on the category of algebras of a regular equational theory. If $\mathcal{S}$ is a semigroup monad, $C$ is a sup-semilattice and $\mathbf{F}$ : $C \rightarrow \mathcal{E} \mathcal{M}(S)$ is a functor, then $\mathbf{F}$ is what is called a strong sup-semilattice decomposition of the semigroup $\bigsqcup_{C}(\mathbf{F})$. Thus such lax morphisms of monads induce additional operations on the categories of Eilenberg-Moore algebras that we shall call (generalized) Płonka sums.

Dually, the natural transformation $\psi_{l}$ is an isomorphism iff $\mathcal{S}$ preserves $C$ indexed limits. Such monads are even more rare. But, again, if a monad $\mathcal{S}$ has some additional good properties, it may happen that for some small categories $C$ the functor $\Pi_{C}: \mathcal{A}^{C} \longrightarrow \mathcal{A}$, the composition of functors

$$
\mathcal{A}^{C} \stackrel{i^{*}}{\longrightarrow} \mathcal{A}^{o b(C)} \stackrel{\prod}{\longrightarrow} \mathcal{A}
$$

induced by functors between small categories $C \stackrel{i}{\longleftarrow} o b(C) \stackrel{!}{\longrightarrow} 1$, can be equipped with a natural transformation $\rho_{C}: \prod_{C} \circ \hat{\mathcal{S}} \longrightarrow \mathcal{S} \circ \prod_{C}$, so that $\left(\prod_{C}, \rho_{C}\right): \hat{\mathcal{S}} \rightarrow \mathcal{S}$ is an oplax morphism of monads. Each such oplax morphism of monads induces in turn an operation on the category of Kleisli algebras

$$
\prod_{C}: \mathcal{K}(\mathcal{S})^{C} \cong \mathcal{K}(\hat{\mathcal{S}}) \longrightarrow \mathcal{K}(\mathcal{S})
$$

By analogy we shall call such operations Płonka products. 
Remark 1 For any ultrafilter $U$ on any set $I$ we have an ultraproduct functor $\operatorname{Set}^{I} \stackrel{[U]}{\longrightarrow}$ Set, see [15] for details. If $\mathcal{T}$ is a finitary monad on $\operatorname{Set}$ and $\hat{\mathcal{S}}$ its lift to $\operatorname{Set}^{I}$, then $[U]: \hat{\mathcal{S}} \longrightarrow \mathcal{S}$ is a strong monad morphism, as a consequence of Łoś theorem. It induces the ultraproduct operation on the category of $\mathcal{S}$-algebras.

\section{Category of Regular Polynomials Over an Algebra}

Let $\mathcal{R}=(\mathcal{R}, \eta, \mu)$ be a semi-analytic monad (c.f. [22]), $R: \mathbb{S} \rightarrow$ Set a functor such that for any set $X$

$$
\mathcal{R}(X)=\sum_{n \in \omega}\left[\begin{array}{l}
X \\
n
\end{array}\right] \otimes_{n} R_{n}
$$

By [22], $R$ is the functor part of a regular operad, i.e. a monoid in the monoidal category $\mathrm{Set}^{\mathbb{S}}$ with the substitution tensor. We define a functor

$$
C P_{r}^{\mathcal{R}}: \mathcal{E} \mathcal{M}(\mathcal{R}) \longrightarrow \text { Cat }
$$

associating to an $\mathcal{R}$-algebra $(A, \alpha: \mathcal{R}(A) \rightarrow A)$ a category of regular polynomials $C P_{r}^{\mathcal{R}}(A, \alpha)$ as follows. The objects of $C P_{r}^{\mathcal{R}}(A, \alpha)$ are elements of $A$. A morphism in $C P_{r}^{\mathcal{R}}(A, \alpha)$ is an equivalence class of triples

$$
[\vec{a}, i, r] \sim: \vec{a}(i) \rightarrow \alpha([\vec{a}, r] \sim)
$$

where $\vec{a}:(n] \rightarrow A$ is an injection, $i \in(n], r \in R_{n}$, for some $n \in \omega$. Note that $[\vec{a}, r] \sim$ is an element of $\mathcal{R}(A)$. We identify triples

$$
\langle\vec{a} \circ \sigma, i, r\rangle \sim\langle\vec{a}, \sigma(i), R(\sigma)(r)\rangle
$$

where $\sigma \in S_{n}$. The identity morphism is

$$
[a, 1, \iota]_{\sim}: a \rightarrow \alpha\left([a, \iota]_{\sim}\right)=a
$$

where $\iota \in R_{1}$ is the unit of the regular operad $R$. The composition is defined by the substitution of regular terms into regular terms possibly with normalization. In detail, the composite of a pair of morphisms

$$
\vec{a}(i) \stackrel{[\vec{a}, i, r]_{\sim} \longrightarrow}{\longrightarrow} \alpha(\vec{a}, r)=\vec{a}^{\prime}(j) \stackrel{\left[\vec{a}^{\prime}, j, r^{\prime}\right]_{\sim}}{\longrightarrow} \alpha\left(\vec{a}^{\prime}, r^{\prime}\right)
$$

is

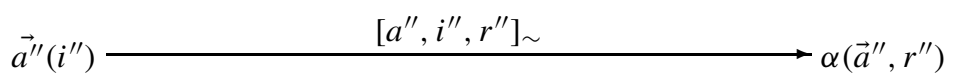

where $r \in R_{n}, r^{\prime} \in R_{m}$; the function $\vec{a}^{\prime \prime}:(k] \rightarrow A$ is the injection part for surjectioninjection factorization $\vec{a}^{\prime \prime} \circ s:(n+m-1] \rightarrow A$ of the function $\vec{a}^{\prime}(j \backslash \vec{a}):(n+m-1] \rightarrow A$ such that

$$
\vec{a}^{\prime}(j \backslash \vec{a})(l)= \begin{cases}\vec{a}^{\prime}(l) & \text { if } 1 \leq l<j \\ \vec{a}(l-j+1) & \text { if } j \leq l<n+j \\ \vec{a}^{\prime}(l-n+1) & \text { if } n+j \leq l<n+m .\end{cases}
$$


Thus $\vec{a}^{\prime}(j \backslash \vec{a})$ replaces $j$ in the domain of $\vec{a}^{\prime}$ by the whole function $\vec{a}$. Such function might not be an injection and $\vec{a}^{\prime \prime}$ is the injection part of it. $i^{\prime \prime}=s(i+j-1) \in(k]$, i.e. $i^{\prime \prime}$ is the element in $(k]$ that correspond to $i \in(n] . r^{\prime \prime}=R(s)\left((\iota, \ldots, r, \ldots, \iota) * r^{\prime}\right)$, i.e. the value under the action of $s:(n+m-] \rightarrow(k]$ on the composition in the regular operad $R$ of $r^{\prime}$ with $r$ placed into the $j$ 's place. This ends the definition of the category $C P_{r}^{\mathcal{R}}(A, \alpha)$.

A homomorphism $h:(A, \alpha) \longrightarrow\left(A^{\prime}, \alpha^{\prime}\right)$ induces a functor

$$
C P_{r}^{\mathcal{R}}(h): C P_{r}^{\mathcal{R}}(A, \alpha) \longrightarrow C P_{r}^{\mathcal{R}}\left(A^{\prime}, \alpha^{\prime}\right)
$$

so that the morphism $[\vec{a}, i, r] \sim: \vec{a}(i) \rightarrow \alpha(\vec{a}, r)$ is sent to

$$
\left[\vec{a}^{\prime}, s(i), R(s)(r)\right]_{\sim}: \vec{a}^{\prime}(s(i))=h(\vec{a}(i)) \rightarrow h(\alpha(\vec{a}, r))=\alpha^{\prime}\left(\vec{a}^{\prime}, R(s)(r)\right)
$$

where $\vec{a}^{\prime} \circ s$ is surjection-injection factorization of $h \circ \vec{a}$.

We note for the record

Fact 4.1 For any semi-analytic monad $\mathcal{R}$, the above construction defines a functor

$$
C P_{r}^{\mathcal{R}}: \mathcal{E} \mathcal{M}(\mathcal{R}) \longrightarrow C a t
$$

associating to $\mathcal{R}$-algebras their categories of regular polynomials.

Remark 2 One can describe the category of polynomials $C P_{r}^{\mathcal{R}}(A, \alpha)$ in terms of the regular equational theory $T_{\mathcal{R}}$ corresponding to the monad $\mathcal{R}$ as follows. Its objects are elements of the algebra $(A, \alpha)$. A morphism from $a$ to $b$ is given by

1. a regular term of the theory $T_{\mathcal{R}}$ in $n$ variable $r\left(x_{1}, \ldots, x_{n}\right)$ (all the variables necessarily explicitly occur in $r$ );

2. a certain variable number $i \in(n]$;

3. an injective function $\vec{a}:(n] \rightarrow A$ interpreting variables occurring in $r\left(x_{1}, \ldots, x_{n}\right)$;

4. the domain of the morphism $a$ is equal to the interpretation of the $i$ 'th variable $\vec{a}(i)$;

5. the codomain of the morphism $b$ is equal to the value of the term $r\left(x_{1}, \ldots, x_{n}\right)$ under the interpretation of the variables $\vec{a}$.

The composition of such morphisms is defined as (the most reasonable) substitution.

The reason why this definition works for semi-analytic monads is, as we explained in the introduction, that in the corresponding regular equational theories there is a good notion of an occurrence of a variable in a term.

If $\tau: \mathcal{R} \rightarrow \mathcal{V}$ is a morphism of semi-analytic monads, then the functors associating categories of regular polynomials to those monads are related as follows. The morphism $\tau$ induces a 'forgetful' functor $\mathcal{E} \mathcal{M}(\tau): \mathcal{E M}(\mathcal{V}) \rightarrow \mathcal{E} \mathcal{M}(\mathcal{R})$ and we have a natural transformation

$$
\gamma^{\tau}: C P_{r}^{\mathcal{R}} \circ \mathcal{E} \mathcal{M}(\tau) \longrightarrow C P_{r}^{\mathcal{V}}: \mathcal{E} \mathcal{M}(\mathcal{V}) \longrightarrow C a t
$$

defined for a $\mathcal{V}$-algebra $(B, \beta)$ a functor

$$
\gamma_{(B, \beta)}^{\tau}: C P_{r}^{\mathcal{R}}(B, \beta \circ \tau) \longrightarrow C P_{r}^{\mathcal{V}}(B, \beta)
$$

constant on object, and sending morphism $[\vec{a}, i, r] \sim: \vec{a}(i) \longrightarrow \beta \circ \tau(\vec{a}, r)$ to the morphism

$$
\left[\vec{a}, i, \tau_{n}(r)\right]_{\sim}: \vec{a}(i) \longrightarrow \beta\left(\vec{a}, \tau_{n}(v)\right)
$$

where $n \in \omega, r \in R_{n}$ and $\tau_{n}: R_{n} \rightarrow V_{n}$. 
Infinitary Case

It should be clear how to define the functor of associating to algebras for a monad $\mathcal{R}$ in $\operatorname{SanMnd}_{\kappa}$, SanMnd $_{\infty}$ or SANMND the category of (suitable infinitary) regular polynomials

$$
C P_{\infty r}^{\mathcal{R}}: \mathcal{E M} \longrightarrow C a t
$$

in analogy with the finitary case. Note that we never consider regular polynomials of arity that exceeds the cardinality of the algebra. The details are left for the reader.

\section{Płonka Sums of Algebras for a Semi-Analytic Monad}

In this section we shall study operations of generalized Płonka sums on the categories of algebras for semi-analytic monad indexed by categories of regular polynomials of algebras for another semi-analytic monads.

Recall that if $(S, \eta, \mu)$ and $\left(T, \eta^{\prime}, \mu^{\prime}\right)$ are monads on categories $C$ and $D$, respectively, then the lax morphism of monads ([2], [4]) $(F, \tau):(S, \eta, \mu) \rightarrow\left(T, \eta^{\prime}, \mu^{\prime}\right)$ is a functor $F: C \rightarrow D$ together with a natural transformation $\tau: T \circ F \rightarrow F \circ S$ satisfying

$$
\tau \circ \eta_{F}^{\prime}=F(\eta), \quad \tau \circ \mu_{F}^{\prime}=F(\mu) \circ \tau_{S} \circ T(\tau)
$$

Let $\pi: \mathcal{R} \rightarrow \mathcal{T}$ be a morphism of semi-analytic monads, defined by a natural transformation (denoted by the same letter) $\pi: R \rightarrow T$ in $\operatorname{Set}^{\mathbb{S}}$. Let $(A, \alpha)$ be a $\mathcal{T}$-algebra. Let us denote the category of regular polynomials $C P_{r}(A, \alpha)$ as $\mathbf{A}$, for short. The monad $\hat{\mathcal{R}}$ is the lift of the monad $\mathcal{R}$ to the category $\operatorname{Set}^{\mathbf{A}}$ (see introduction for details of the definition of $\hat{\mathcal{R}})$. We shall define a lax morphism of monads induced by $\pi$ and $\mathcal{T}$-algebra $(A, \alpha)$

$$
\left(\bigsqcup_{(A, \alpha)}, \lambda^{\pi,(A, \alpha)}\right): \hat{\mathcal{R}} \rightarrow \mathcal{R}
$$

We usually drop superscripts $(A, \alpha)$ and write $\mathcal{R}$ instead of $\pi$ in $\lambda^{\pi,(A, \alpha)}$ when it does not lead to a confusion. We also write $\bigsqcup_{A}$ rather than $\bigsqcup_{(A, \alpha)}$. Let $\mathbf{F}: \mathbf{A} \rightarrow \mathcal{E} \mathcal{M}(\mathcal{R})$ be a functor, and $F: \mathbf{A} \rightarrow$ Set the composition of $\mathbf{F}$ with the forgetful functor. We shall define the component

$$
\lambda_{F}^{\mathcal{R}}: \mathcal{R}\left(\coprod_{a \in A} F(a)\right) \longrightarrow \coprod_{a \in A} \mathcal{R}(F(a))
$$

of the natural transformation $\lambda^{\mathcal{R}}$. Let $[\vec{x}, r]_{\sim} \in \mathcal{R}\left(\coprod_{a \in A} F(a)\right)$, where $\vec{x}:(n] \rightarrow$ $\bigsqcup_{a \in A} F(a)$ is an injection, $r \in R_{n}$. Let $p: \bigsqcup_{a \in A} F(a) \rightarrow A$ be the projection from the coproduct to the index set. Let $\vec{a} \circ s$ be a surjection-injection factorization of $p \circ \vec{x}$ as in the diagram below. Moreover, we have a unique function $\vec{x}^{\prime}$ making the triangle in the middle commute, where $\kappa_{a}: F(a) \rightarrow \bigsqcup_{a \in A} F(a)$ is the injection into coproduct. To explain the right hand square in the diagram below, note that $[\vec{a}, R(s)(r)] \in \mathcal{R}(A)$ and hence

$$
\pi([\vec{a}, R(s)(r)])=\left[\vec{a}, \pi_{m}(R(s)(r))\right] \in \mathcal{T}(A)
$$

We put

$$
b=\alpha\left(\left[\vec{a}, \pi_{m}(R(s)(r))\right]\right)
$$


and, for $i \in(n]$, we have a morphism

$$
\psi_{i}=\left[\vec{a}, s(i), \pi_{m}(R(s)(r))\right]: \vec{a}(s(i)) \longrightarrow b
$$

in the category A. The morphism $\vec{z} \circ g$ is a surjection-injection factorization of $\left[F\left(\psi_{i}\right)\right]_{i \in(n]^{\circ}}$ $\vec{x}^{\prime}$. This explains the construction of the diagram

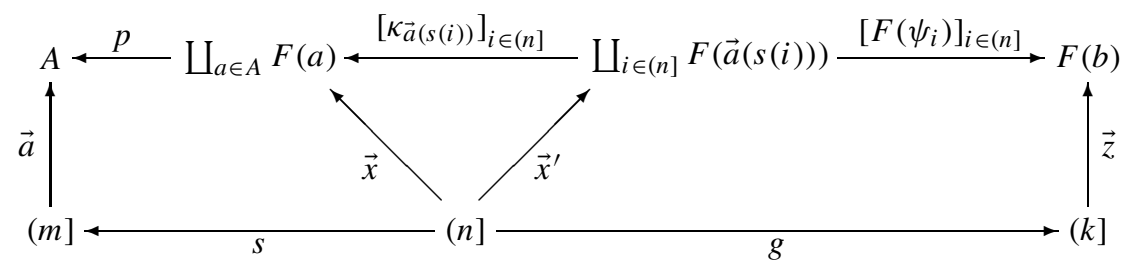

Finally, we put

$$
\lambda_{F}^{\mathcal{R}}([\vec{x}, r] \sim)=[\vec{z}, R(g)(r)] \sim
$$

A simple verification shows

Proposition $5.1\left(\bigsqcup_{A}, \lambda^{\mathcal{R}}\right): \hat{\mathcal{R}} \rightarrow \mathcal{R}$ is a lax morphism of monads.

Lifting the above morphism of monads, we obtain $\mathcal{T}$-indexed Płonka sum of $\mathcal{R}$-algebras, i.e. for any $\mathcal{T}$-algebra $(A, \alpha)$ we obtain an operation

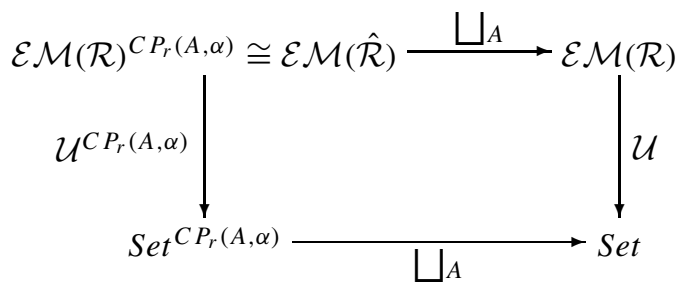

The following proposition explains how Płonka sums interact with lax morphisms of semi-analytic monads.

\section{Proposition 5.2 Let}

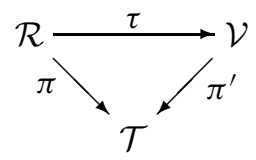

be a commuting triangle in the category of semi-analytic monads, $(A, \alpha)$ a $\mathcal{T}$-algebra. Then we have a commuting square

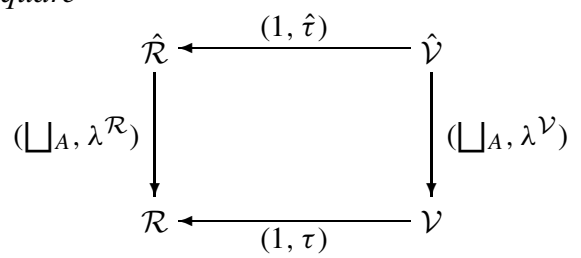

of lax morphisms of monads, where transformations $\lambda^{\mathcal{R}}$ and $\lambda^{\mathcal{V}}$ are induced by morphism $\pi$ and $\pi^{\prime}$, respectively. 
Proof Let us fix a $\mathcal{T}$-algebra $(A, \alpha)$ and $\mathbf{A}=C P_{r}(A, \alpha)$. We shall denote the natural transformations in $S e t^{\mathbb{S}}$ that give rise to $\tau, \pi, \pi^{\prime}$ by the same letters. We need to show that the square of functors and natural transformations in $\operatorname{Nat}\left(\operatorname{Set}^{\mathbf{A}}, \operatorname{Set}\right)$

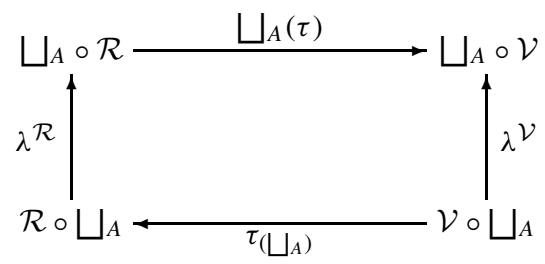

commutes.

Let $F: \mathbf{A} \rightarrow$ Set be a functor. An element $[\vec{x}, r] \sim$ of $\mathcal{R} \circ \bigsqcup_{A}(F)$ is represented by a pair $\langle\vec{x}, r\rangle$ such that for some $n \in \omega, \vec{x}:(n] \rightarrow \bigsqcup_{a \in A} F(a)$ is an injection and $r \in R_{n}$. Let $\vec{a}, \mathrm{~s}, \vec{x}^{\prime}, b, \psi_{i}, g, \vec{z}$ be as in the definition of $\lambda_{F}^{\mathcal{R}}\left([\vec{x}, r]_{\sim}\right)$ above. Thus

$$
\bigsqcup_{A}(\tau)\left(\lambda^{\mathcal{R}}([\vec{x}, r] \sim)\right)=\tau_{F(b)}([\vec{z}, R(g)(r)] \sim)=\left[\vec{z}, \tau_{m}(R(g)(r))\right] \sim
$$

As $\tau$ is a natural transformation and $\pi^{\prime} \circ \tau=\pi$, for $i \in(n]$, the morphism $\psi_{i}$ in $\mathbf{A}$ is equal to the morphism

$$
\psi_{i}^{\prime}=\left[\vec{a}, s(i), \pi_{m}^{\prime}\left(V(s)\left(\tau_{n}(r)\right)\right)\right]_{\sim}: \vec{a}(s(i)) \longrightarrow b
$$

Thus

$$
\left.\lambda^{\mathcal{V}}\left(\tau_{\left(\bigsqcup_{A}\right)}\left([\vec{x}, r]_{\sim}\right)\right)=\lambda^{\mathcal{V}}\left(\left[\vec{x}, \tau_{n}(r)\right]_{\sim}\right)\right)=\left[\vec{z}, V(s)\left(\tau_{n}(r)\right)\right]_{\sim}
$$

Now from (3) and (4) it follows that the square (2) commutes.

Lifting Proposition 5.2 to the categories of Eilenberg-Moore algebras, with the notation as above, we obtain that regular interpretations of regular theories induce morphisms of algebras that preserve Płonka sums. Thus we have

Corollary 5.3 The functor between categories of algebras $\mathcal{E} \mathcal{M}(\tau): \mathcal{E} \mathcal{M}(\mathcal{V}) \rightarrow \mathcal{E} \mathcal{M}(\mathcal{R})$ induced by the semi-analytic natural transformation $\tau: \mathcal{R} \rightarrow \mathcal{T}$ preserves $\mathcal{T}$-indexed Ptonka sum, i.e. for any $\mathcal{T}$-algebra $(A, \alpha)$ the square

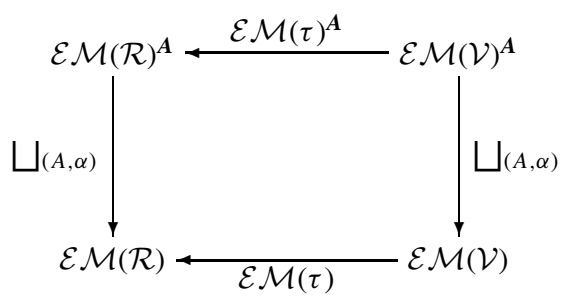

commutes, up to a canonical isomorphism, where $\boldsymbol{A}=C P_{r}(A, \alpha)$.

From Proposition 5.2 we also have 


\section{Corollary 5.4 Let}

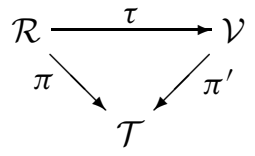

be a commuting triangle in the category of semi-analytic monads, $(A, \alpha)$ a $\mathcal{T}$-algebra $\boldsymbol{A}=$ $C P_{r}(A, \alpha)$. Let $\boldsymbol{F}: \boldsymbol{A} \rightarrow \mathcal{E} \mathcal{M}(\mathcal{V})$ be a functor, $F=\mathcal{U}^{\mathcal{V}} \circ \boldsymbol{F}: \boldsymbol{A} \rightarrow$ Set. Then the two $\mathcal{R}$-algebra structures on $\bigsqcup_{a \in A} F(a)$

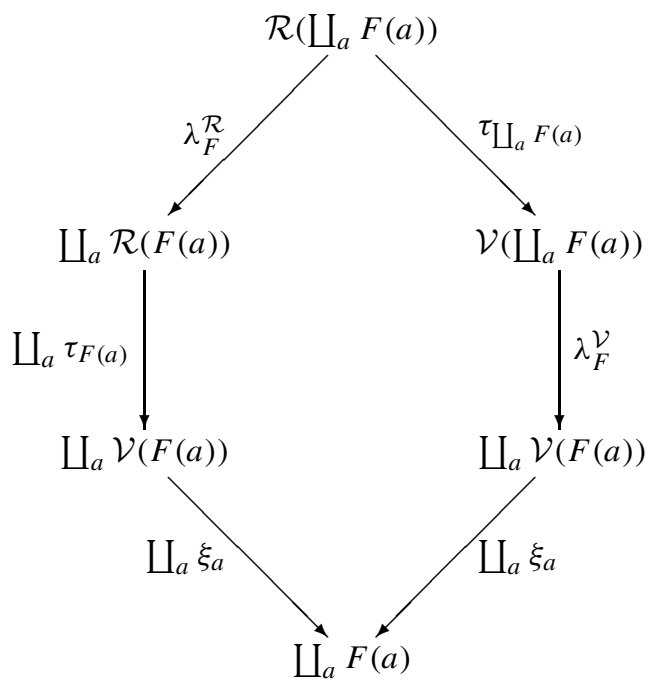

coincide.

In order to make these sums independent of a given monad $\mathcal{T}$ and particular morphisms $\pi$ and $\pi^{\prime}$, we can take as $\mathcal{T}$ the terminal semi-analytic monad, that is, the monad $\mathcal{L}$ of supsemilattices. In such a way we have equipped any category of algebras for a semi-analytic monad $\mathcal{R}$ with a canonical system of Płonka sums indexed by the categories of regular polynomials over sup-semilattices. By Proposition 5.1, the semi-analytic morphism of semianalytic monads induces a functor between categories of algebras that preserves Płonka sums. In fact, this property characterizes semi-analytic morphisms of semi-analytic monads, i.e. the converse of Corollary 5.4, and hence also Proposition 5.1, is also true. We have

Theorem 2.1 Let $\tau: \mathcal{R} \rightarrow \mathcal{V}$ be an arbitrary lax morphism of monads between semianalytic monads. Then $\tau$ is a semi-cartesian iff the induced functor $\mathcal{E} \mathcal{M}(\tau)$ between categories of algebras preserves Ptonka sums.

Proof The 'only if' part is just Proposition 5.2.

To see the converse, for a lax morphism of monads $\tau: \mathcal{R} \rightarrow \mathcal{V}$ we shall define a natural transformation $\sigma: R \rightarrow V$ such that $\hat{\sigma}=\tau$. Let us fix $r \in R_{n}, n \in \omega$. Then $\left[1_{(n]}, r\right] \sim \in \mathcal{R}(n]$ and we have

$$
\tau_{(n]}\left(\left[1_{(n]}, r\right] \sim\right)=[\vec{x}, v]_{\sim} \in \mathcal{V}(n]
$$


where $\vec{x}:(m] \rightarrow(n]$ is an injection and $v \in V_{m}$, for some $m \in \omega$. To end the proof

1. we show that $m=n$ and hence $\vec{x}$ is a permutation;

2. we put $\sigma_{n}(r)=V(\vec{x})(r)$ and show that $\sigma: R \rightarrow V$ so defined is a natural transformation;

3. finally we show that $\hat{\sigma}=\tau$.

Let $X \subseteq(n]$ be the image of $\vec{x}$ is $(n]$. Let $\mathbf{F}: \mathcal{P}(n] \longrightarrow \mathcal{E} \mathcal{M}(\mathcal{V})$ be a functor sending $y \in \mathcal{P}(n]$ to the one-element algebra $\left(\{Y\}, \xi_{Y}\right)$. Thus we have a commuting diagram

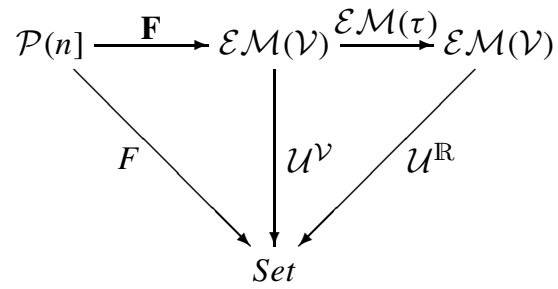

with $F$ being an inclusion functor. Note that $\bigsqcup_{Y \in \mathcal{P}(n]}\{Y\}=\mathcal{P}(n]$.

Let $\{-\}:(n] \rightarrow \mathcal{P}(n]$ be the singleton morphism i.e. $\{-\}(i)=\{i\}$, for $i \in(n]$. Since $\mathcal{E} \mathcal{M}(\tau)$ preserves Płonka sums and $\tau$ is a natural transformation, we have a commuting diagram

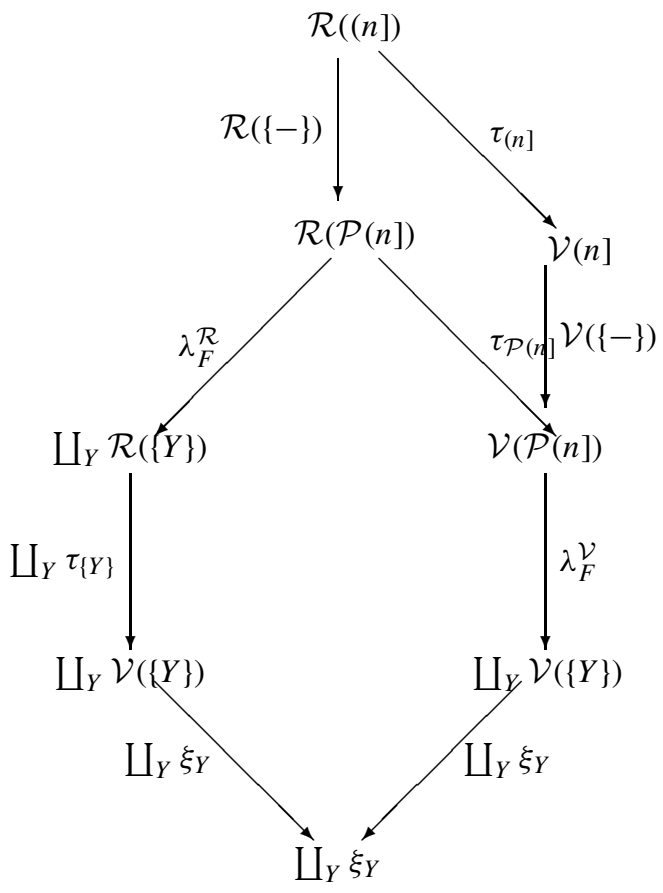

Evaluating two morphisms at $\left[1_{(n]}, r\right] \sim \in\left[\begin{array}{c}(n] \\ n\end{array}\right] \otimes_{n} R_{n} \subseteq \mathcal{R}(n]$ after some calculations, we get

$$
\coprod_{Y}\left(\xi_{Y} \circ \tau_{\{Y\}}\right) \circ \lambda_{F}^{\mathcal{R}} \circ \mathcal{R}(\{-\})\left(\left[1_{(n]}, r\right] \sim\right)=(n]
$$


and

$$
\left.\left(\coprod_{Y} \xi_{Y}\right) \circ \lambda \mathcal{V}_{F}^{\mathcal{V}} \circ \tau_{\mathcal{P}(n]} \circ \mathcal{R}(\{-\})\left(\left[1_{(n]}, r\right]\right]_{\sim}\right)=\left(\coprod_{Y} \xi_{Y}\right) \circ \lambda \mathcal{V}_{F}^{\mathcal{V}} \circ \mathcal{V}(\{-\})\left([\vec{x}, v]_{\sim}\right)=X
$$

Thus $X=(n]$ and hence $n=m$, as required.

The verification of the naturality of $\sigma: R \rightarrow V$ we leave for the reader. We end by showing that $\hat{\sigma}=\tau$.

By definition of $\sigma$, we have for $n \in \omega$ and $r \in R_{n}$

$$
\tau_{(n]}\left(\left[1_{(n]}, r\right]_{\sim}\right)=\left[1_{(n]}, \sigma_{n}(r)\right]_{\sim}=\hat{\sigma}\left(\left[1_{(n]}, r\right]_{\sim}\right)
$$

Thus for any $[\vec{z}, r]_{\sim} \in\left[\begin{array}{l}Z \\ n\end{array}\right] \otimes_{n} R_{n} \subseteq \mathcal{R}(Z)$ we have

$$
\begin{gathered}
\tau_{Z}\left([\vec{z}, r]_{\sim}\right)=\tau_{Z}\left(\mathcal{R}(\vec{z})\left[1_{(n]}, r\right]_{\sim}\right)= \\
=\mathcal{V}(\vec{z})\left(\tau_{(n]}\left(\left[1_{(n]}, r\right]_{\sim}\right)\right)=\mathcal{V}(\vec{z})\left(\hat{\sigma}\left(\left[1_{(n]}, r\right]_{\sim}\right)\right)= \\
=\hat{\sigma}_{Z}\left(\mathcal{R}(\vec{z})\left(\left[1_{(n]}, r\right]_{\sim}\right)\right)=\hat{\sigma}_{Z}\left([\vec{z}, r]_{\sim}\right)
\end{gathered}
$$

i.e. $\tau=\hat{\sigma}$.

\section{Remark 3}

1. If $(A, \alpha)$ is an $\mathcal{L}$-algebra, i.e. a sup-semilattice, then the posetal collapse of the category $C P_{r}(A, \alpha)$ is $(A, \leq)$ i.e. the partial order on the set $A$ determined by the sup-lattice structure $(A, \alpha)$. Clearly, we have a canonical functor $C P_{r}(A, \alpha) \rightarrow(A, \leq)$. The original definition of Płonka sum on $\mathcal{R}$-algebras is defined on functors $F: C P_{r}(A, \alpha) \rightarrow$ $\mathcal{E} \mathcal{M}(\mathcal{R})$ that factorize through $(A, \leq)$.

2. The inclusion of the category of semi-analytic monads into finitary monads on Set has a right adjoint. This means that any finitary monad $\mathcal{M}$ on Set has its 'regular part' given by

$$
\operatorname{reg}(\mathcal{M})(X)=\sum_{n \in \omega}\left[\begin{array}{l}
X \\
n
\end{array}\right] \otimes_{n} \mathcal{M}(n]
$$

Thus we have a canonical functor $\mathcal{E} \mathcal{M}(\mathcal{M}) \longrightarrow \mathcal{E} \mathcal{M}(\operatorname{reg}(\mathcal{M}))$ induced by $\operatorname{reg}(\mathcal{M}) \rightarrow \mathcal{M}$. Hence any functor $F: C \rightarrow \mathcal{E} \mathcal{M}(\mathcal{M})$ extends to a functor $F: C \rightarrow \mathcal{E} \mathcal{M}(\operatorname{reg}(\mathcal{M}))$. Thus we can compute Płonka sum of algebras for any monad except that the resulting algebra will satisfy only the regular equations of the theory.

Infinitary Case

The terminal monads in SANMND is the powerset monad, i.e. the monad for suplattices. We can redo the whole story above in the infinitary case getting the analogous results. We just note for the record one

Theorem 5.6 Let $\tau: \mathcal{R} \rightarrow \mathcal{V}$ be an arbitrary lax morphism of monads between monads in SANMND. Then $\tau$ is semi-cartesian iff the induced functor $\mathcal{E} \mathcal{M}(\tau): \mathcal{E} \mathcal{M}(\mathcal{V}) \rightarrow \mathcal{E} \mathcal{M}(\mathcal{R})$ between categories of algebras preserves Ptonka sums.

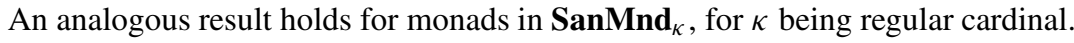




\section{Category of Linear Polynomials Over an Algebra}

In this and the next sections we shall describe a parallel story to the one described in the previous two sections for analytic monads. We shall concentrate on the subtle differences pertinent in this case. As we saw in Section 2 analytic monads are more specific than semianalytic ones. This has the following consequences:

1. In this context, we have not only a notion of a variable that occurs in a term as in the regular case but we can now count occurrences; this is why we can and we will take more subtle category of polynomials, namely the category of linear polynomials.

2. The terminal analytic monad is the monad for the commutative monoids; thus we shall equip (canonically) any category of algebra for an analytic monad with operations of Płonka sums whose arities are categories of linear polynomials over commutative monoids.

3. As the series expansion of analytic functors are simpler than the expansions of semianalytic ones, the definition of the morphism of monads (natural transformation part) inducing Płonka sums is simpler. But then the characterization results are analogous.

Let $\mathcal{A}=(\mathcal{A}, \eta, \mu)$ be an analytic monad, $A: \mathbb{B} \rightarrow$ Set a functor such that for set $X$

$$
\mathcal{A}(X)=\sum_{n \in \omega} X^{n} \otimes_{n} A_{n}
$$

$A$ is the functor part of a symmetric operad, i.e. a monoid in the monoidal category $S e t^{\mathbb{B}}$ with the substitution tensor. We define a functor

$$
C P_{l}^{\mathcal{A}}: \mathcal{E} \mathcal{M}(\mathcal{A}) \longrightarrow C a t
$$

associating to an $\mathcal{A}$-algebra $(X, \xi: \mathcal{A}(X) \rightarrow X)$ a category of linear polynomials $C P_{l}^{\mathcal{A}}(X, \xi)$ as follows. The objects of $C P_{l}^{\mathcal{A}}(X, \xi)$ are elements of $X$. A morphism in $C P_{l}^{\mathcal{A}}(X, \xi)$ is an equivalence class of triples

$$
[\vec{x}, i, a]_{\sim}: \vec{x}(i) \rightarrow \alpha(\vec{x}, a)
$$

where, for some $n \in \omega, \vec{x}:(n] \rightarrow X$ is a function, $i \in(n], a \in A_{n}$. We identify triples

$$
\langle\vec{x} \circ \sigma, i, a\rangle \sim\langle\vec{x}, \sigma(i), A(\sigma)(a)\rangle
$$

where $\sigma \in S_{n}$. The identity morphism is

$$
[x, 1, \iota] \sim: x \rightarrow \xi(x, \iota)=x
$$

where $\iota \in A_{1}$ is the unit of the symmetric operad $A$. The composition is defined by the substitution of linear-regular terms into linear-regular terms (normalization is not needed). In detail, it is defined as follows

$$
\vec{x}(i) \underset{[\vec{x}, i, a]_{\sim}}{\stackrel{\left[x^{\prime \prime}, i^{\prime \prime}, a^{\prime \prime}\right]_{\sim}}{\longrightarrow} \xi(\vec{x}, a)=\vec{x}^{\prime}(j) \underset{\left[\vec{x}^{\prime}, j, a^{\prime}\right]_{\sim}}{\longrightarrow}} \xi\left(\vec{x}^{\prime}, a^{\prime}\right)
$$

where $a \in A_{n}, a^{\prime} \in A_{n}$; the function $\vec{x}^{\prime \prime}=\vec{x}^{\prime}(j \backslash \vec{x}):(n+m-1] \rightarrow X$ is defined so that

$$
\vec{x}^{\prime}(j \backslash \vec{x})(l)= \begin{cases}\vec{x}^{\prime}(l) & \text { if } 1 \leq l<j \\ \vec{x}(l-j+1) & \text { if } j \leq l<n+j \\ \vec{x}^{\prime}(l-n+1) & \text { if } n+j \leq l<n+m .\end{cases}
$$


Thus $\vec{x}^{\prime}(j \backslash \vec{x})$ replaces $j$ in the domain of $\vec{x}^{\prime}$ by the whole function $\vec{x} . a^{\prime \prime}=$ $\left((\iota, \ldots, a, \ldots, \iota) * a^{\prime}\right)$, i.e. the composition in the symmetric operad $A$ of $a^{\prime}$ with $a$ placed into the $j$ 's place. This ends the definition of the category $C P_{l}^{\mathcal{A}}(X, \xi)$.

A homomorphism $h:(X, \xi) \longrightarrow\left(X^{\prime}, \xi^{\prime}\right)$ induces a functor

$$
C P_{l}^{\mathcal{A}}(h): C P_{l}^{\mathcal{A}}(X, \xi) \longrightarrow C P_{l}^{\mathcal{A}}\left(X^{\prime}, \xi^{\prime}\right)
$$

so that the morphism $[\vec{x}, i, a] \sim: \vec{x}(i) \rightarrow \xi(\vec{x}, a)$ is sent to

$$
\left[h \circ \vec{x}^{\prime}, i, a\right] \sim: h\left(\vec{x}^{\prime}(i)\right) \rightarrow h(\xi(\vec{x}, a))=\alpha^{\prime}\left(\vec{x}^{\prime}, a\right)
$$

We note for the record

Fact 6.1 For any analytic monad $\mathcal{A}$, the construction above defines a functor

$$
C P_{l}^{\mathcal{R}}: \mathcal{E M}(\mathcal{A}) \longrightarrow \text { Cat }
$$

associating to $\mathcal{A}$-algebras their categories of linear polynomials.

Remark 4 One can describe the category of polynomials $C P_{l}^{\mathcal{R}}(X, \xi)$ as follows. Its objects are elements of the algebra $(X, \xi)$. A morphism from $x$ to $y$ can be described in a similar way as in the semi-analytic case as follows:

1. a linear-regular term in $n$ variable $a\left(x_{1}, \ldots, x_{n}\right)$ (all the variables necessarily explicitly occur in $a$ exactly once);

2. a certain variable number $i \in(n]$ is chosen;

3. a function $\vec{x}:(n] \rightarrow X$ interpreting variables occurring in $a\left(x_{1}, \ldots, x_{n}\right)$;

4. the domain on the morphism $x$ is equal to the interpretation of the $i$-th variable i.e. $\vec{x}(i)$;

5. the codomain of the morphism $y$ is equal to the value of the term $a\left(x_{1}, \ldots, x_{n}\right)$ under the interpretation of the variables $\vec{x}$.

The composition of such morphisms is defined as (the most reasonable) substitution.

The reason why this definition works for analytic monads is, as we explained in the introduction, that in the corresponding linear-regular equational theories there is a good notion of the number of occurrences of a variable in a term.

Note that the category of linear polynomials of a monoid, as well as the category of regular polynomials of sup-semilattices, have natural structure of a monoidal category with multiplication (or sup) playing the role of the tensor.

If $\tau: \mathcal{A} \rightarrow \mathcal{A}^{\prime}$ is a morphism of analytic monads, then the functors associating categories of linear polynomials to those monads are related as follows. The morphism $\tau$ induces a 'forgetful' functor $\mathcal{E} \mathcal{M}(\tau): \mathcal{E} \mathcal{M}\left(\mathcal{A}^{\prime}\right) \rightarrow \mathcal{E} \mathcal{M}(\mathcal{A})$ and we have a natural transformation

$$
\gamma^{\tau}: C P_{l}^{\mathcal{A}} \circ \mathcal{E} \mathcal{M}(\tau) \longrightarrow C P_{l}^{\mathcal{A}^{\prime}}: \mathcal{E} \mathcal{M}\left(\mathcal{A}^{\prime}\right) \longrightarrow \text { Cat }
$$

defined for a $\mathcal{A}^{\prime}$-algebra $(X, \xi)$ a functor

$$
\gamma_{(X, \xi)}^{\tau}: C P_{l}^{\mathcal{A}}(X, \xi \circ \tau) \longrightarrow C P_{l}^{\mathcal{A}^{\prime}}(X, \xi)
$$

constant on object, and sending morphism $[\vec{x}, i, a]_{\sim}: \vec{x}(i) \longrightarrow \xi \circ \tau(\vec{x}, a)$ to the morphism

$$
\left[\vec{x}, i, \tau_{n}(a)\right]_{\sim}: \vec{x}(i) \longrightarrow \xi\left(\vec{x}, \tau_{n}\left(a^{\prime}\right)\right)
$$

where $a \in A_{n}$ and $\tau_{n}: A_{n} \rightarrow A_{n}^{\prime}$. 


\section{Płonka Sums of Algebras for an Analytic Monad}

The theory of Płonka sums for the categories of algebras for analytic monads is parallel to the theory of Płonka sums for the categories of algebras for semi-analytic monads described in Section 5. We need to replace categories of regular polynomials by the categories of linear polynomials and the terminal semi-analytic monad of sup-semilattices by the terminal analytic monad of commutative monoids. As the details are very similar in both cases, we shall give the basic definition and state some of the facts without proofs leaving the verification as an exercise.

Let $\pi: \mathcal{A} \rightarrow \mathcal{T}$ be a morphism of analytic monads, defined by a natural transformation (denoted by the same letter) $\tau: A \rightarrow T$ in $\operatorname{Set}^{\mathbb{B}}$. Let $(Z, \zeta)$ be a $\mathcal{T}$-algebra. Let denote the category of the linear polynomials $C P_{l}(Z, \zeta)$ as $\mathbf{Z}$. The monad $\hat{\mathcal{A}}$ is a lift of the $\operatorname{monad} \mathcal{A}$ to the category $\operatorname{Set}^{\mathbf{Z}}$. We shall define a lax morphism of monads induced by $\pi$ and $\mathcal{R}$-algebra $(Z, \zeta)$

$$
\left(\bigsqcup_{(Z, \zeta)}, \lambda^{\mathcal{A},(Z, \zeta)}\right): \hat{\mathcal{A}} \rightarrow \mathcal{A}
$$

We usually drop superscripts $(Z, \zeta)$ and write $\mathcal{A}$ instead of $\pi$ in $\lambda^{\pi,(Z, \zeta)}$ when it does not lead to a confusion. We write $\bigsqcup_{Z}$ rather than $\bigsqcup_{(Z, \zeta)}$. Let $F: \mathbf{Z} \rightarrow$ Set be a functor. We shall define

$$
\lambda_{F}^{\mathcal{A}}: \mathcal{A}\left(\coprod_{z \in Z} F(z)\right) \longrightarrow \coprod_{z \in Z} \mathcal{A}(F(z))
$$

as

$$
[\vec{x}, a] \sim \mapsto\left[f \circ \vec{x}^{\prime}, a\right] \sim \in \mathcal{A}(F(b))
$$

where $\vec{x}^{\prime}$ is a lift of $\vec{x}$ as before, the function $f$ and element $b \in Z$ will be described below and are displayed in the diagram below. Since $[\vec{x}, a] \sim \in \mathcal{A}\left(\coprod_{z \in Z} F(z)\right)$, it follows that for some $n \in \omega, \vec{x}:(n] \rightarrow \bigsqcup_{z \in Z} F(z)$ is a function and $a \in A_{n}$. Let $p: \bigsqcup_{z \in Z} F(z) \rightarrow Z$ be the projection from the coproduct to the index set. We put

$$
\left.b=\zeta\left(\left[p \circ \vec{x}, \pi_{n}(a)\right)\right]\right)
$$

and, for $i \in(n]$, we have a morphism

$$
\psi_{i}=\left[p \circ \vec{x}, i, \pi_{n}(a)\right]: \vec{x}(i) \longrightarrow b
$$

in the category $\mathbf{Z}$. The function $f$ is

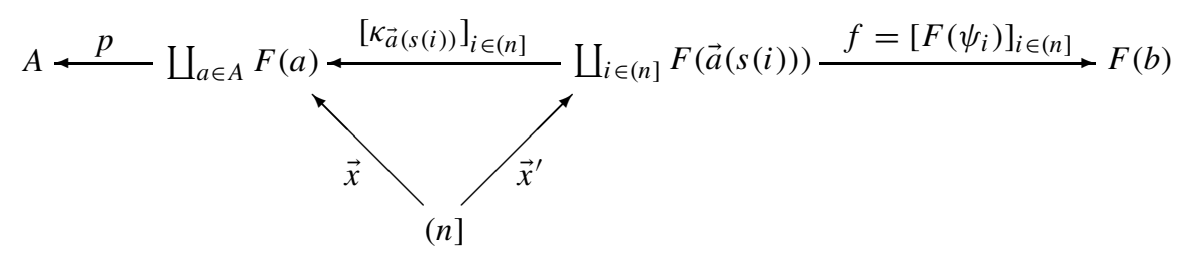

A simple verification shows

Proposition 7.1 $\left(\bigsqcup_{A}, \lambda^{\mathcal{A}}\right): \hat{\mathcal{A}} \rightarrow \mathcal{A}$ is a lax morphism of monads. 
Lifting the above morphism of monads, we obtain $\mathcal{T}$-indexed Płonka sum of $\mathcal{R}$-algebras, i.e. for any $\mathcal{T}$-algebra $(A, \alpha)$ we obtain an operation

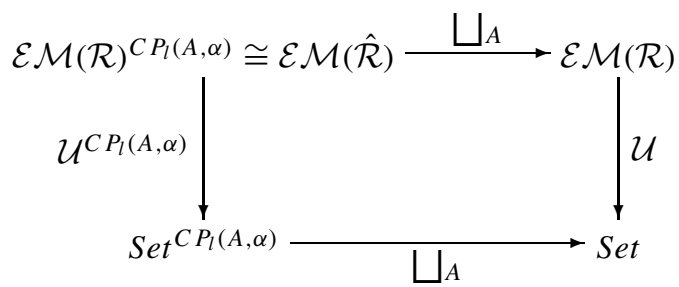

The analog of Proposition 5.2 holds verbatim for the lax morphisms of analytic monads. Thus again in this case, whenever we have $\pi: \mathcal{A} \rightarrow \mathcal{T}$ a lax morphism of analytic monads, we have operations of Płonka sums on the category of algebras for $\mathcal{A}$ whose arities are the categories of linear polynomials over algebras for $\mathcal{T}$. As the category of analytic monads has the terminal object, the monad $\mathcal{C}$ for commutative monoids, we can make these sums independent of a varying analytic monad $\mathcal{T}$. We equip any category of algebras for an analytic monad $\mathcal{A}$ with a canonical system of Płonka sums with arities being the categories of linear polynomials over commutative monoids (i.e. algebras for the terminal analytic $\operatorname{monad} \mathcal{C}$ ). We have

Theorem 7.2 Let $\tau: \mathcal{A} \rightarrow \mathcal{A}^{\prime}$ be an arbitrary lax morphism of monads between analytic monads. Then $\tau$ is weakly cartesian iff the induced functor $\mathcal{E} \mathcal{M}(\tau)$ between categories of algebras preserves Płonka sums.

\section{Some Examples}

1. Let $\tau: \mathcal{R} \rightarrow \mathcal{T}$ be a morphism of semi-analytic monads. $N$ is a $\mathcal{R}$-algebra, $M$ is a $\mathcal{T}$-algebra. Then Płonka sum is the usual binary product of algebras

$$
\bigsqcup_{c \in C} \mathbf{F}(c) \cong N \times \operatorname{Alg}(\tau)(M)
$$

where $\mathbf{F}: C=C P_{r}(M) \longrightarrow A \lg (S)$ is a constant functor with value $N$. For analytic monads the analogous fact holds true.

2. Let $h: M \rightarrow N$ be a homomorphism of $\mathcal{R}$-algebras for a semi-analytic monad $\mathcal{R}$. Then on the coproduct in Set of universes of $M$ and $N$ there is a structure $\mathcal{R}$-algebra, the Płonka sum over the 2-element sup-semilattice. The constants are interpreted in $M$. The operations are interpreted in $M$ if all arguments are in $M$, and in $N$ after transferring the necessary arguments from $M$ to $N$ by $h$, otherwise.

3. The following is a more elaborate example of a Płonka sum. It is inspired by the conversations I had with F.W. Lawvere after my talk in Coimbra during the Workshop on Category Theory in honor of George Janelidze, on the occasion of his 60th birthday.

(a) Theory $\mathrm{Mat}_{R}$ is the theory whose operations from $n$ to $m$ are $m \times n$-matrices of elements of a rig $R$. Neither theory of rigs nor $M_{a} t_{R}$ are regular theories, however the affine part of $M a t_{R}$ is a regular theory of convexity algebras denoted $C V$. It has one constant say $\perp$ and those operations $\left\langle r_{1}, \ldots, r_{n}\right\rangle: n \rightarrow 1$ that $\sum_{i=1}^{n} r_{i}=1$, and $r_{i} \neq 0$ for $i=1, \ldots, n$.

(b) Now let us fix $R$ to be the rig of non-negative real numbers. Free algebra for $C V$ on $n$ generators is an $n$-dimensional simplex (with one distinguished vertex $\perp$ ). 
(c) Fix any set $Z$. Let $\Omega$ denote the category of regular polynomials over the supsemilattice of finite subsets $\mathcal{P}_{<\omega}(Z)$. There is an obvious forgetful functor $U$ : $\Omega \rightarrow$ Set sending the morphism $\left[i, \sum_{j=1}^{n} r_{j} x_{j}, X_{1}, \ldots, X_{n}\right]: X_{i} \rightarrow \bigcup_{j} X_{j}$ in $\Omega$ to the inclusion $X_{i} \rightarrow \bigcup_{j} X_{j}$. The functor $\mathbf{F}: \Omega \rightarrow A \lg (C V)$ is the composition of $U$ with the free $C V$-algebra functor $\mathcal{F}: \operatorname{Set} \rightarrow \operatorname{Alg}(C V)$.

(d) The Płonka sum $\bigsqcup_{\Omega} \mathbf{F}$ is the disjoint sum of simplices spanned by $\perp$ and finite subsets of $Z$. The $k$-ary operations $O_{\vec{r}}$ given by an $k$-tuple $\vec{r}=\left\langle r_{1}, \ldots, r_{k}\right\rangle$ such that $r_{i} \neq 0$ and $\sum_{i}^{k} r_{i}=1$ in $\bigsqcup_{\Omega} \mathbf{F}$ is defined as follows. Let $y_{i} \in \Delta_{X_{i}}$ be elements of $\bigsqcup_{\Omega} \mathbf{F}$, i.e. $y_{i}=\sum_{j \in X_{i} \cup\{\perp\}} s_{j}^{i} \cdot x_{j}^{i}$ for $i=1, \ldots, k$, and some $s_{j}^{i} \in R$. Then

$$
O_{\vec{r}}\left(y_{1}, \ldots, y_{k}\right)=\sum_{i=1}^{k} r_{i} \sum_{j \in X_{i} \cup\{\perp\}} r_{j}^{i} \cdot x_{j}^{i}
$$

and it is clearly an element of $\Delta_{\bigcup_{i}} X_{i}$.

4. There are some similarities between Płonka sums and graded rings. However there are differences, as well. A graded ring is build from a (lax) monoidal functor from a monoid $M$ considered as a discrete monoidal category to abelian groups. For example the graded ring of polynomials in $n$ variables $\mathbb{Z}\left[x_{1}, \ldots, x_{1}\right]$ arises in this way from the functor $P: \omega \rightarrow A b$ sending $n$ to the set of uniform polynomials of degree $n$, say $P_{n}$. The coherence transformations for this monoidal functor are multiplications $\varphi_{n, m}: P_{n} \times P_{m} \longrightarrow P_{n+m}$ and identity $\bar{\varphi}: \mathbb{Z} \rightarrow \mathbb{Z}=P_{0}$.

\section{Płonka Products}

The Płonka products are not as common as Ponka sums but they exists in the literature in a different setup. We describe this briefly below.

Let $(\mathcal{S}, \eta, \mu)$ be a monad on $\operatorname{Set},(\hat{\mathcal{S}}, \hat{\eta}, \hat{\mu})$ be a lift of this monad on $\operatorname{Set}^{2}$, and $\mathbb{1}$ be the unique monad on the terminal category $\mathbf{1} \cong \operatorname{Set}^{0}$. Clearly the category $\operatorname{Set}$ with binary products and the terminal object is a monoidal category $($ Set $, \times, 1, \alpha, \lambda, \varrho)$. Let $\varphi: \times \circ$ $\hat{\mathcal{S}} \longrightarrow \mathcal{S} \circ \times$ and $\bar{\varphi}: 1 \rightarrow \mathcal{S}(1)$ be two natural transformations.

Recall that $\mathbf{M N D}_{\text {lax }}$ denotes the 2-category of monads, lax morphisms of monads, and transformations of lax morphisms of monads. This category has finite products.

Then we have

Proposition 9.1 With the notation as above

1. If $(\mathcal{S}, \varphi, \bar{\varphi}, \eta, \mu)$ is a monoidal monad on (Set, $\times, 1, \alpha, \lambda, \varrho)$, then

$$
(\times, \phi): \hat{\mathcal{S}} \rightarrow \mathcal{S} \quad \text { and } \quad(1, \bar{\varphi}): \mathbb{1} \rightarrow \mathcal{S}
$$

are oplax morphism of monads and hence they induce Ptonka products

$$
\prod_{I}: \mathcal{K}(\mathcal{S})^{2} \rightarrow \mathcal{K}(\mathcal{S}) \quad \text { and } \quad \prod_{\emptyset}: \mathbf{1} \rightarrow \mathcal{K}(\mathcal{S})
$$

2. On the other hand, if $(\times, \phi)$ and $(1, \bar{\varphi})$ are oplax morphism of monads so that together with $(\alpha, \lambda, \varrho)$ constitute a monoidal category object on $(\mathcal{S}, \eta, \mu)$ in $\mathbf{M N D}_{\text {lax }}$, then $(\mathcal{S}, \varphi, \bar{\varphi}, \mu, \eta, \mu)$ is an oplax monoidal monad on $(\operatorname{Set}, \times, 1, \alpha, \lambda, \varrho)$ and $\mathcal{K}(\mathcal{S})$ is the Kleisli object for this monad in $\mathbf{M N D}_{\text {lax }}$. 
Remark 5 From the proposition above it follows that if $\mathcal{S}$ is a commutative monad (c.f [5, 10]) then, for any finite set $I$, we have an oplax morphism of monads $\left(\prod_{I}, \psi\right): \mathcal{S}^{I} \rightarrow \mathcal{S}$ (where $\mathcal{S}^{I}$ is a lift of $\mathcal{S}$ to $S e t^{I}$ ) and a Płonka product $\mathcal{K}(S)^{I} \rightarrow \mathcal{K}(S)(\hat{\mathcal{S}}$ is the lift of $\mathcal{S}$ to $\left.\operatorname{Set}^{I}\right)$.

Note that the (finitary) commutative monads on Set form a full reflective subcategory of Mnd.

\section{Distributive Laws}

There is yet another property that the terminal monads in AnMnd and SanMnd have in common: they distribute over all the other monads in 'their' respective categories.

The sup-semilattice monad $\mathcal{L}$ lifts canonically to the category of algebras for any regular monad due to the fact that it distributes over any semi-analytic monad $\mathcal{R}$ and the commutative monoid monad $\mathcal{C}$ lifts canonically to the category of algebras for any analytic monad due to the fact that $\mathcal{C}$ distributes over any analytic monad $\mathcal{A}$. The former statement belongs to the folklore and the latter might be new. We shall give precise formulas describing these distributive laws.

The theory of sup-lattices has exactly one regular operation of every arity $n \in \omega$. To fix the notation we denote such an $n$-ary operation as $\mu_{n}$. The coefficient functor $L: \mathbb{S} \rightarrow$ Set of the corresponding semi-analytic monad $\mathcal{L}$ is the terminal object $\operatorname{Set}^{\mathbb{S}}$, so that $L(n)=$ $\left\{\mu_{n}\right\} . \mathcal{L}$ is the value of the right adjoint to the inclusion functor SanMnd $\rightarrow$ Mnd on the terminal monad in Mnd. If $\mathcal{R}$ is another semi-analytic monad with the coefficient functor $R: \mathbb{S} \rightarrow$ Set, then we define the distributive law of $\mathcal{L}$ over $\mathcal{R}$

$$
\rho_{\mathcal{R}}: \mathcal{R} \circ \mathcal{L} \longrightarrow \mathcal{L} \circ \mathcal{R}
$$

as follows. Let $R L, L R: \mathbb{S} \rightarrow$ Set be the coefficient functors of the composition functors $\mathcal{R} \circ \mathcal{L}$ and $\mathcal{L} \circ \mathcal{R}$, respectively. Fix a set $X$ and an element $\left[\vec{x} ; \phi ; \mu_{m_{1}}, \ldots, \mu_{m_{k}} ; r\right] \sim$ in $\left[\begin{array}{l}X \\ n\end{array}\right] \otimes_{n} R L_{n}$, where $\vec{x}:(n] \rightarrow X$ is an injection, $\phi:(m] \rightarrow(n]$ is a surjection, $m=$ $\sum_{i=1}^{k} m_{i}$ and $r \in R_{k}$. We put

$$
\rho_{\mathcal{R}, X}\left(\left[\vec{x} ; \phi ; \mu_{m_{1}}, \ldots, \mu_{m_{k}} ; r\right] \sim\right)=\left[\vec{y} ; \psi ; r, \ldots, r ; \mu_{M}\right]_{\sim} \in\left[\begin{array}{c}
X \\
k \cdot M
\end{array}\right] \otimes_{k \cdot M} L R_{k \cdot M}
$$

where $r$ occurs on the right side $M=m_{1} \cdot m_{2} \cdot \ldots \cdot m_{k}$ times, $\psi:(k \cdot M] \rightarrow\left(n^{\prime}\right]$, $\vec{y}:\left(n^{\prime}\right] \rightarrow X$ is a surjective-injective factorization of the function

$$
f:(k \cdot M] \cong(k] \times\left(m_{1}\right] \times \ldots \times\left(m_{k}\right] \longrightarrow X
$$

such that for $\left\langle i, j_{1}, \ldots, j_{k}\right\rangle \in(k] \times\left(m_{1}\right] \times \ldots \times\left(m_{k}\right]$

$$
f\left(i, j_{1}, \ldots, j_{k}\right)=\phi_{i}\left(j_{i}\right)
$$

and $\phi_{i}\left(m_{i}\right] \rightarrow X$ is the composition of $\phi$ with the obvious inclusion $\left(m_{i}\right] \rightarrow(m]$.

Since we allow in the above definition $m_{i}$ to be equal 0 , so $M$ can be equal 0 independently of the values of other $m_{i}$ 's. This is why $\rho_{\mathcal{R}}$ does not need to be semi-analytic natural transformation and hence $\mathcal{L} \circ \mathcal{R}$ does not need to be a semi-analytic monad. The situation can be improved from that point of view if we drop the constant in $\mathcal{L}$, i.e. if we look at the (regular) theory of sup-semilattices without bottom element i.e. the monad $\mathcal{L}^{\prime}$ whose 
coefficient functor $L^{\prime}: \mathbb{S} \rightarrow$ Set is as $L$ except that $L^{\prime}(\emptyset)=\emptyset$. Then the above formulas do define a distributive law

$$
\rho_{\mathcal{R}}^{\prime}: \mathcal{R} \circ \mathcal{L}^{\prime} \longrightarrow \mathcal{L}^{\prime} \circ \mathcal{R}
$$

which is semi-analytic and hence the monad $\mathcal{L}^{\prime} \circ \mathcal{R}$ is semi-analytic. Note that $\mathcal{L}^{\prime}$ is the value of the right adjoint to the inclusion functor SanMnd $\rightarrow$ Mnd on the only proper submonad of the terminal monad in Mnd, corresponding to the theory without any operations and having one equation $x_{1}=x_{2}$.

As we mentioned above, the commutative monoid monad $\mathcal{C}$ distributes over any analytic monad $\mathcal{A}$. Again, the theory of commutative monoids is the theory that has a unique analytic (this time) operation $\mu_{n}$ of arity $n$, for every $n \in \omega$. The coefficient functor $C: \mathbb{B} \rightarrow$ Set is the terminal object in $\mathrm{Set}^{\mathbb{B}}$. The distributive law

$$
\alpha_{\mathcal{A}}: \mathcal{A} \circ \mathcal{C} \longrightarrow \mathcal{C} \circ \mathcal{A}
$$

is even simpler to define then $\rho_{\mathcal{R}}$ for semi-analytic monads. Fix a set $X$. For $\left[\vec{x} ; \mu_{n_{1}}, \ldots, \mu_{n_{k}} ; a\right] \sim$ in $X^{n} \otimes_{n} A C_{n}$ we put

$$
\alpha_{\mathcal{A}, X}\left(\left[\vec{x} ; \mu_{n_{1}}, \ldots, \mu_{n_{k}} ; a\right] \sim\right)=\left[\vec{y} ; a, \ldots, a ; \mu_{N}\right] \sim
$$

where $n=\sum_{i=1}^{k} n_{i}, a$ occurs on the right side $N=n_{1} \cdot n_{2} \cdot \ldots \cdot n_{k}$ times, and the function $\vec{y}:(k \cdot N] \rightarrow X$ is defined for $\left\langle i, j_{1}, \ldots, j_{k}\right\rangle \in(k] \times\left(n_{1}\right] \times \ldots \times\left(n_{k}\right]$

$$
\vec{y}\left(i, j_{1}, \ldots, j_{k}\right)=\vec{x}_{i}\left(j_{i}\right)
$$

and $\vec{x}_{i}:\left(n_{i}\right] \rightarrow X$ is the composition of $\vec{x}$ with the obvious inclusion $\left(n_{i}\right] \rightarrow(n]$. Again the distributive law $\alpha_{\mathcal{A}}$ is neither analytic nor even semi-analytic. If we consider the submonad of $\mathcal{C}$ without the constant i.e. the monad for the commutative semigroups $\mathcal{C}^{\prime}$, then we shall get a semi-analytic distributive law

$$
\alpha_{\mathcal{A}}^{\prime}: \mathcal{A} \circ \mathcal{C}^{\prime} \longrightarrow \mathcal{C}^{\prime} \circ \mathcal{A}
$$

given by essentially the same formulas as $\alpha_{\mathcal{A}}$. Thus the monad $\mathcal{C}^{\prime} \circ \mathcal{A}$ is semi-analytic but not analytic in general.

The verification of the above statements is easy but a bit tedious. We state it for the record.

\section{Theorem 10.1}

1. The monads $\mathcal{L}$ and $\mathcal{L}^{\prime}$ for sup-semilattices and for sup-semilattices without bottom distributes over any other semi-analytic monad $\mathcal{R}$ in a canonical way through the distributive laws $\rho_{\mathcal{R}}$ and $\rho_{\mathcal{R}}^{\prime}$, respectively. The distributive law $\rho_{\mathcal{R}}^{\prime}: \mathcal{R} \circ \mathcal{L}^{\prime} \longrightarrow \mathcal{L}^{\prime} \circ \mathcal{R}$ is semi-analytic and the composed monad $\mathcal{L}^{\prime} \circ \mathcal{R}$ is again semi-analytic.

2. The monads $\mathcal{C}$ and $\mathcal{C}^{\prime}$ for commutative monoids and for commutative semigroups distribute over any other analytic monad $\mathcal{A}$ in a canonical way through the distributive laws $\alpha_{\mathcal{R}}$ and $\alpha_{\mathcal{A}}^{\prime}$, respectively. The distributive law $\alpha_{\mathcal{A}}^{\prime}: \mathcal{A} \circ \mathcal{C}^{\prime} \longrightarrow \mathcal{C}^{\prime} \circ \mathcal{A}$ is semi-analytic and the composed monad $\mathcal{C}^{\prime} \circ \mathcal{A}$ is semi-analytic.

The extension of these result to the infinitary cases we leave for the reader.

Acknowledgments The author want to thank the anonymous referee for useful suggestions concerning presentation of the paper.

Open Access This article is distributed under the terms of the Creative Commons Attribution License which permits any use, distribution, and reproduction in any medium, provided the original author(s) and the source are credited. 


\section{References}

1. Adamek, J., Velebil, J.: Analytic functors and weak pullbacks. Theory Appl. Categ 21(11), 191-209 (2008)

2. Beck, J.: Distributive laws. Lect. Notes Math. 80, 119-140 (1969)

3. Bénabou, J.: Catégories avec multiplication. C.R. Acad. Sci. Paris 256, 1887-1890 (1963)

4. Barr, M., Wells, C.: Toposes, Triples and Theories. Grundl. Math. Wissenschaften no. 278, SpringerVerlag (1985)

5. Coumans, D., Jacobs, B.: Scalars, Monads, and Categories. (2011) arXiv.1003.0585v2 [math.RA]

6. Dolinka, I., Vinčić, M.: Involutory Płonka sums. Period. Math. Hung. 46(1), 17-31 (2003)

7. Graczyńska, E., Pastijn, F.: A generalization of Płonka sums. Fundam. Math. 120, 53-62 (1984)

8. Janelidze, G., Laan, V., Márki, L.: Limit preservation properties of the greatest semilattice image functor. Int. J. Algebra Comput. 18, 853-867 (2008)

9. Joyal, A.: Foncteurs analytiques et espéces de structures. In: Lecture Notes in Mathematics, vol. 1234, pp. 126-159. Springer (1986)

10. Kock, A.: Commutative monads, Monads on symmetric monoidal closed categories. Arch. Math. 21, 1-10 (1970)

11. Leinster, T.: Higher Operads, Higher Categories, London Mathematical Society, Lecture Note Series. Cambridge University Press, Cambridge (2004) (math.CT/0305049)

12. Łoś, J.: Quelques remarques, théorémes et problèmes sur les classes définissables d'algèbres, Mathematical Interpretation of Formal Systems, pp. 98-113. North-Holland Publishing Co., Amsterdam (1955)

13. MacLane, S.: Categories for the working Mathematician. Graduate Text in Mathematics, 2nd edn. Springer (1998)

14. MacLane, S.: Natural associativity and commutativity. Rice Univ. Stud. 49, 28-46 (1963)

15. Makkai, M.: Ultraproducts and categorical logic. In: Di Prosco, C. (ed.) Proceedings, Caracas 1983, Methods in Categorical Logic, Lecture Notes in Mathematics, vol. 1130, pp. 222-309. Springer-Verlag (1985)

16. Makkai, M.: Stone duality for first order categorical logic. Adv. Math. 65, 97-170 (1987)

17. Makkai, M.: Duality and definability in first order logic, Memoirs of AMS 503 (1993)

18. Manes, E.G.: Implementing collection classes with monads. Math. Struct. Comput. Sci. 8, 231-276 (1998)

19. Płonka, J.: On a method of construction of abstract algebras. Fundam. Math. 61, 191-198 (1967)

20. Street, R.: The formal theory of monads. J. Pure Appl. Algebra 2, 149-168 (1972)

21. Szawiel, S., Zawadowski, M.: Theories of analytic monads. Accepted in Math. Struct. in Comput. Sci. (2013)

22. Szawiel, S., Zawadowski, M.: Monads of regular theories. Appl. Categ. Struct. (2013). doi:10.1007/ s10485-013-9331-x

23. Tamura, T.: The theory of construction of finite semigroups, I. Osaka Math. J. 8, 243-261 (1956)

24. Zawadowski, M.: Descent and duality. Ann. Pure Appl. Logic 71(2), 131-188 (1995) 